\title{
弹塑性粗粗表面实际接触面积演变规律研究 ${ }^{*}$
}

\author{
刘检华 张飞凯丁晓宇 \\ (北京理工大学机械与车辆学院 北京 100081)
}

\begin{abstract}
摘要: 粗粘表面的实际接触面积直接影响精密机电设备配合表面的摩擦因数、热导/电导率、接触应力等。然而, 针对弹塑性 接触行为进行系统性地研究很少, 弹塑性粗粘表面实际接触面积的演变规律尚不明确。针对这一问题, 通过理论研究获得了 弹塑性粗糙表面实际接触面积的影响因素, 并引入弹塑性接触力学数值计算方法, 对具有不同材料参数和表面形貌特征的弹 塑性粗精表面的接触行为进行数值仿真计算, 得到各种情况下的表面实际接触面积随着平均接触压力的变化曲线, 总结出了 实际接触面积演变规律与这些影响因素之间的映射关系, 并最终得到实际接触面积的计算公式, 为弹塑性接触力学的相关工 程应用奠定了基础。
\end{abstract}

关键词: 接触力学; 弹塑性; 粗糙表面; 实际接触面积; 接触数值计算

中图分类号: TB42

\section{Study on the Evolution of Real Contact Area between Elastic-plastic Rough Surfaces}

\author{
LIU Jianhua ZHANG Feikai DING Xiaoyu \\ (School of Mechanical Engineering, Beijing Institute of Technology, Beijing 100081)
}

\begin{abstract}
The real contact area of elastic-plastic rough surfaces significantly affects the friction coefficient, the thermal/electrical conductivity, and the contact stress of the assembly surfaces of precision electromechanical devices. However, as far as the authors known, the elastic-plastic contact behavior is rarely studied systematically, and the evolution of the real contact area has not been investigated. To solve this problem, the factors affecting the real contact area of elastic-plastic rough surfaces are revealed by theoretical research. Based on this, the contact behavior of various elastic-plastic rough surfaces with different material and surface topography is numerically studied, and real contact area versus average contact pressure curves are obtained. With these results, the quantitative relationship between the evolution of the real contact area and the factors is presented, which will provide fundamental theory for scientists and engineers working in this field.
\end{abstract}

Key words: contact mechanics; elastic-plastic; rough surface; real contact area; numerical contact analysis

\section{0 前言}

工程中，宏观光滑的机械结构表面在微观尺度 上都是粗粘不平的。使用表面轮廓测量仪等设备观 测这些表面时, 会发现这些表面具有高低不平的波 峰波谷。在精密机电设备的装配过程中，在法向装 配接触压力的作用下, 两个机械加工的微观粗䊁配 合面的波峰部分首先发生接触, 波谷之间仍然会存

* 国家自然科学基金资助项目(51935003)。20200625 收到初稿, 20210120 收到修改稿
在大量的接触间隙。实际接触区域的面积所占整个 配合表面宏观名义接触面积的比例很小, 这个比例 称为接触率(或无量纲实际接触面积, 简称实际接触 面积)。实际接触面积的大小与法向载荷、表面微观 形貌、材料力学性能等参数相关, 并直接影响精密 机电设备配合表面的摩擦因数、热导/电导率、接触 应力等。粗糙表面实际接触面积演变规律研究、尤 其是弹塑性粗粘表面实际接触面积演变规律研究, 具有重要的学术价值和工程意义。

粗糙表面接触力学研究起始于 1881 年 HERTZ 提出的 Hertz 接触理论 ${ }^{[1]}$, 在该理论的基础上, 
GREENWOOD 和 WILLIAMSON ${ }^{[2]}$ 在 1966 年率先 提出了一种多微凸体接触计算模型 (GreenwoodWilliamson 模型, 简称 G-W 模型)。在此基础上, 有学者对 G-W 模型进行扩展与改进, 提出了很多新 的微凸体模型 ${ }^{[3-12]}$ 。微凸体模型具有计算速度快, 轻载条件计算精度高的特点, 但是它将实际粗精表 面简化为由高度随机或指数分布的球形或其它形状 的微凸体构成的表面, 因此会为模型引入了误差, 使得这一类模型只有在轻载条件下 ${ }^{[13]}$ 具有较好的 计算精度。为了避免这一问题, PERSSON 在 2001 年基于分形理论提出了另外一种不对粗粘表面进行 近似处理的纯弹性粗粘表面接触计算方法 ${ }^{[14-15]}$, 这 种方法被称为 Persson 理论。很多学者通过使用微 凸体模型和 Persson 理论等对轻载的无摩擦的纯弹 性粗糙表面接触问题进行研究发现 ${ }^{[14-16]}$ : 纯弹性粗 粮表面的实际接触面积与平均接触压力近似成正比 例关系, 且比例系数与材料的弹性模量、泊松比以 及表面梯度的均方根之间的定量关系。最新研究结 果表明, 实际接触面积不仅与上述参数相关, 而且 可能与表面的 Nayak 参数 ${ }^{[17]}$ 存在一定的联系。对于 弹塑性粗糙表面的接触问题, 虽然也有部分学者通 过研究发现其实际接触面积随着平均接触压力线性 增加 ${ }^{[7-9]}$, 但是尚未有学者对其进行系统性的研究, 弹塑性粗䊁表面实际接触面积演变规律及其影响因 素尚不明确。

随着计算机技术的提高和数值计算方法的发 展, 数值计算方法逐渐被应用到接触力学领域, 成 为一种常用的接触力学研究方法。接触力学数值计 算方法包括边界元模型(Boundary element method, $\mathrm{BEM})^{[18-22]}$, 半解析法 (Semi-Analytical) ${ }^{[23-34]}$ 和有限 元模型(Finite element method, FEM) ${ }^{[35-45]}$ 等。使用 上述数值计算方法, BORRI-BRUNETTO 等 ${ }^{[36]}$ 和 HYUN 等 ${ }^{[37-38]}$ 分别对具有不同泊松比、表面粗䊁度 等参数的粗糙表面接触问题进行了研究, 也得到实际 接触面积和接触压力近似成正比例关系的结论。在弹 塑性接触力学领域, 虽然 YAN 等 ${ }^{[46]}$, GAO 等 ${ }^{[4]} 、 \mathrm{PEI}$ 等 ${ }^{[39]}$ 、YASTREBOV 等 ${ }^{[42]}$ 和 NELIAS 等 ${ }^{[28]}$ 通过使用 数值计算方法研究发现, 相对于纯弹性粗糙表面, 弹 塑性粗糙表面的实际接触面积与平均接触压力更加 接近正比例关系; 但是同样尚未有学者针对弹塑性 粗糙表面的接触行为进行系统性的研究, 其实际接 触面积演变规律及其影响因素尚不明确。

针对这一问题, 本文首先通过理论研究, 确定 了弹塑性粗䊁表面(理想弹塑性)实际接触面积的影 响因素。然后对具有不同材料参数和表面形貌特征
的弹塑性粗䊁表面的接触行为进行数值仿真计算, 得到各种情况下的表面实际接触面积随着平均接触 压力的变化曲线。最终得出弹塑性粗粘表面实际接 触面积演变规律与这些因素之间的映射关系, 并总 结得到实际接触面积的计算公式。

\section{1 弹塑性粗䊁表面实际接触面积演 变规律}

\section{1 弹塑性粗糙表面实际接触面积演变规律的影 响因素}

当弹塑性粗粘表面受压接触时, 在任意平均接 触压力下, 表面塑性接触区域相对弹性接触区域的 比例为定值。GREENWOOD 和 WILLIAMSON ${ }^{[2]}$ 定 义了塑性指数来描述弹塑性粗粘表面的塑性接触区 域相对弹性接触区域的比例, 而影响塑性指数的参 数为材料的等效弹性模量、硬度(或屈服强度)和表 面梯度的均方根。使用多微凸体模型 ${ }^{[48-49]}$ (例如 BGT 模型)和 Persson 理论 ${ }^{[14-15]}$ 对纯弹性粗䊁表面的实际 接触面积演变规律进行研究都能够得出结论: 当两 个纯弹性粗䊁表面受很小的接触压力载荷时, 实际 接触面积与平均接触压力近似成正比例关系, 其比 例系数 $K$ 仅与材料的弹性模量、泊松比以及表面梯 度的均方根有关。而根据 Tabor 的理论 ${ }^{[50]}$ 可知: 当 塑性表面(理想刚塑性材料)与刚性平面受压接触 时, 无论是否考虑材料塑性变形的不可压缩特性, 材料的硬度(或屈服强度)都只是唯一影响接触面积 与平均接触压力之间的关系的因素。

因此, 在弹塑性接触中, 综合考虑弹性接触区 域、塑性接触区域以及两区域所占的比例, 我们认 为影响弹塑性粗䊁表面实际接触面积演变规律的主 要因素有三个: 材料的等效弹性模量、硬度(或屈服 强度)和表面梯度的均方根。

\section{2 弹塑性粗糙表面接触力学数值计算模型}

为了探究上述三个因素对实际接触面积演变规 律的影响, 需要对具有不同参数类型的弹塑性粗粘 表面与刚性平面的接触问题进行接触建模和数值计 算。当如图 1 所示的无摩擦理想弹塑性粗粘表面与 刚性平面受压接触时, 系统总是趋向于最稳定的状 态即总余位能最小的状态 ${ }^{[1-52]}$ 。因此, 计算粗楉表 面的接触问题等价于求解系统最小势能的问题, 即 最小势能理论

$$
\begin{array}{r}
\min V^{*}(x, y)=\frac{1}{2} \int_{\Omega} p(x, y) u(x, y) \mathrm{d} \Omega+ \\
\int_{\Omega} p(x, y)\left[g_{0}(x, y)-\delta\right] \mathrm{d} \Omega
\end{array}
$$


式中, $V^{*}$ 表示系统的总余位能, $\Omega$ 表示粗䊁表面的 范围, $p(x, y)$ 表示表面接触压力分布, $u(x, y)$ 表示 粗糙表面的法向形变量, $\delta$ 表示两个表面的相对刚 体位移, 可由式(2)求得

$$
\delta=\text { Average }\left[g_{0}(x, y)+u(x, y)\right]_{\text {Contact region }}
$$

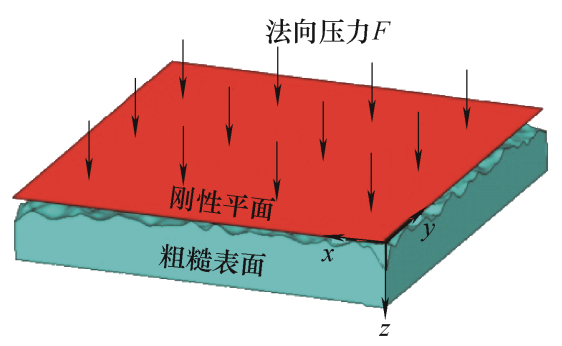

(a) 三维示意图

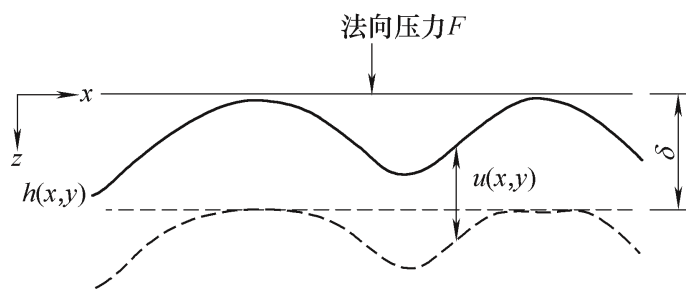

(b) 截面轮廊示意图

图 1 弹塑性粗粘表面与刚性平面接触

$g_{0}(x, y)$ 表示粗粘面与平面的初始接触间隙分 布, 可由式(3)计算得到

$$
g_{0}(x, y)=h(x, y)-0
$$

式中, $h(x, y)$ 为粗楉面的表面高度, 假设粗䊁面与 平面的初始间隙大于等于 0 , 且刚性平面的初始高 度为 0 。同时, 在接触过程中, 接触压力和接触间 隙需要满足以下约束条件

$$
\begin{gathered}
p(x, y) \leqslant H \\
p(x, y) \geqslant 0 \\
g(x, y) \geqslant 0 \\
p(x, y) \cdot g(x, y)=0
\end{gathered}
$$

式中, $H$ 为粗鉌表面材料的维氏硬度, 接触间隙 $g(x, y)=g_{0}(x, y)+u(x, y)-\delta$ 。相对纯弹性边界元 算法, 这里额外增加了约束条件 $p(x, y) \leqslant H$, 以限 制接触压力不能大于材料的维氏硬度。

根据 BOUSSINESQ 理论 ${ }^{[33]}$ 可知, 表面形变与 表面压力分布相关

$$
\begin{gathered}
u\left(x_{a}, y_{a}\right)=\iint G\left(x_{a}-x, y_{a}-y\right) p(x, y) \mathrm{d} x \mathrm{~d} y= \\
G\left(x_{a}, y_{a}\right) * p\left(x_{a}, y_{a}\right)
\end{gathered}
$$

式中, “*” 为卷积运算符, $G(x, y)$ 表示弹性半无
限体表面某点上所受的法向集中力载荷对原点法向 位移的影响系数, 其表达式为

$$
G(x, y)=\frac{1-v^{2}}{\pi E \sqrt{x^{2}+y^{2}}}
$$

式中, $E$ 为材料的弹性模量; $v$ 为材料的泊松比。

综上所述可知, 式(1)仅有一个因变量 $V^{*}(x, y)$ 和一个自变量 $p(x, y)$, 且自变量受约束条件式(4) 限制, 所以弹塑性粗粘面与刚性平面的接触问题的 实质是二次泛函条件极值问题。为了实现二次泛函 条件极值问题的数值求解, 需要对粗糙表面接触问 题进行离散处理。如图 2 所示, 将粗鋉表面离散为 $M \times N$ 点阵, $x, y$ 方向的点间距分别为 $\Delta x, \Delta y$, 点 $(\alpha, \beta)$ 处的表面高度表示为 $h^{\alpha, \beta}=h\left(x_{\alpha}, y_{\beta}\right)$ 、接触 压力表示为 $p^{\alpha, \beta}=p\left(x_{\alpha}, y_{\beta}\right), \alpha, \beta=0,1,2, \cdots, N-1$ 。 式(1)和(5)的离散形式分别为式(7)和(8)。

$$
\begin{aligned}
\min V^{*}= & \frac{1}{2} \sum_{\alpha=0}^{M-1} \sum_{\beta=0}^{N-1}\left[p^{\alpha, \beta} u^{\alpha, \beta}\right]+ \\
& \sum_{\alpha=0}^{M-1} \sum_{\beta=0}^{N-1}\left[p^{\alpha, \beta}\left(g_{0}^{\alpha, \beta}-\delta\right)\right] \\
u^{\alpha, \beta} & =\sum_{\zeta=0}^{M-1} \sum_{\eta=0}^{N-1} K_{i j}^{\alpha-\zeta, \beta-\eta} p^{\zeta \eta}
\end{aligned}
$$

式中, $K_{i j}^{\alpha-\zeta, \beta-\eta}$ 为 $G\left(x_{\alpha}-x_{\xi}, y_{\beta}-y_{\eta}\right)$ 的离散形式, 其近似解为 ${ }^{[54-56]}$

$$
\begin{aligned}
K^{\alpha-\zeta, \beta-\eta} / \frac{1-v^{2}}{\pi E}= & y_{+} \lg \left[x_{+}+\sqrt{{x_{+}{ }^{2}+y_{+}^{2}}^{2}}\right]+ \\
& x_{+} \lg \left[y_{+}+\sqrt{x_{+}^{2}+y_{+}^{2}}\right]- \\
& y_{+} \lg \left[x_{-}+\sqrt{x_{-}^{2}+y_{+}^{2}}\right]- \\
& x_{-} \lg \left[y_{+}+\sqrt{x_{-}^{2}+y_{+}^{2}}\right]- \\
& y_{-} \lg \left[x_{+}+\sqrt{x_{+}^{2}+y_{-}^{2}}\right]- \\
& x_{+} \lg \left[y_{-}+\sqrt{x_{+}^{2}+y_{-}^{2}}\right]+ \\
& y_{-} \lg \left[x_{-}+\sqrt{x_{-}^{2}+y_{-}^{2}}\right]+ \\
& x_{-} \lg \left[y_{-}+\sqrt{x_{-}^{2}+y_{-}^{2}}\right]
\end{aligned}
$$

式中, $x_{+}=x_{\alpha}-x_{\zeta}+\Delta x / 2, x_{-}=x_{\alpha}-x_{\zeta}-\Delta x / 2$, $y_{+}=y_{\beta}-y_{\eta}+\Delta y / 2, \quad y_{-}=y_{\beta}-y_{\eta}-\Delta y / 2$ 。

同时, 式(7)需要满足约束条件

$$
\begin{gathered}
p^{\alpha, \beta} \leqslant H, \\
p^{\alpha, \beta} \geqslant 0, \\
g^{\alpha, \beta} \geqslant 0, \\
p^{\alpha, \beta} g^{\alpha, \beta}=0
\end{gathered}
$$



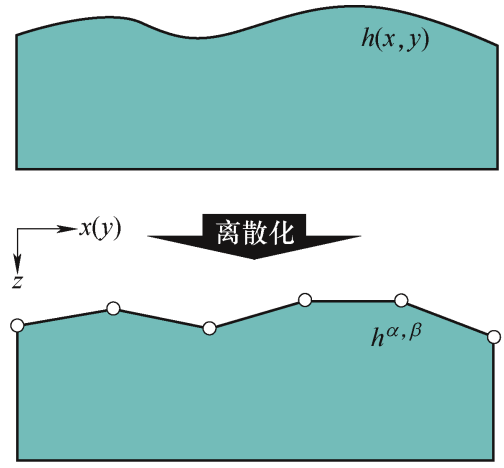

(a) 截面示意图

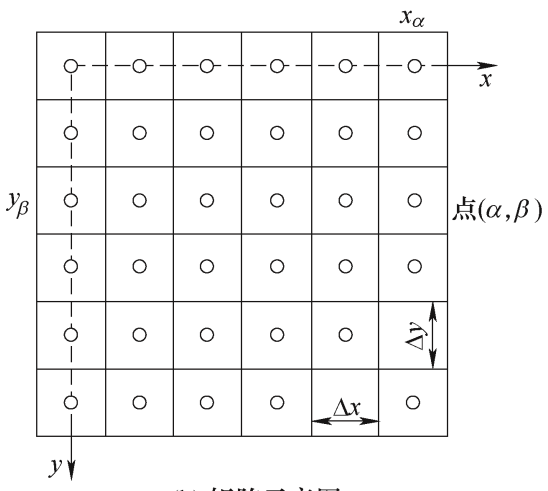

(b) 矩阵示意图

图 2 粗楉表面的离散化处理

为了更快速地求解式(7)所示的二次泛函在式 (10)所示的约束下的条件极值, 本文采用共轭梯度 法加速迭代收玫速度 ${ }^{[55,57]}$ 。同时, 在数值计算过程 中, 采用矩阵运算对式(8)进行求解会更加方便快 捷, 该式的矩阵形式为

$$
\boldsymbol{u}=\boldsymbol{K} * \boldsymbol{p}
$$

式中, $\boldsymbol{u} 、 \boldsymbol{K}$ 和 $\boldsymbol{p}$ 都是大小为 $M \times N$ 的矩阵, $*$ 表 示卷积运算。对矩阵进行线性卷积的数值计算需要 $(M \cdot N)^{2}$ 次乘法运算, 如此庞大的计算量极大地限 制了数值求解的计算效率。因此, JU 等 ${ }^{[58]}$ 首次引入 快速傅里叶变换(Fast Fourier transform, FFT)求解接 触问题的线性卷积求解，基于 FFT 的卷积计算大幅 度降低了接触问题的计算量。使用快速傅里叶变换 求解卷积的公式如下所示

$$
\boldsymbol{u}=\operatorname{IFFT}[\operatorname{FFT}(\boldsymbol{K}) \cdot \operatorname{FFT}(\boldsymbol{p})]
$$

为了避免快速傅里叶变换求解卷积为计算物理 域的边界引入较大的边界混叠误差, 这里采用 WANG 等 ${ }^{[57-59]}$ 提出的 DC-FFT 计算方法, 提高卷积 运算的计算效率，该方法的主要流程如下。

(1) 将大小为 $M \times N$ 的粗粘表面压力分布矩阵 $\boldsymbol{p}$ 扩充为大小为 $(2 M-1) \times(2 N-1)$ 的矩阵 $\boldsymbol{p}_{n e w}$, 扩充区域 用 0 填充。

(2) 在 $(2 M-1) \times(2 N-1)$ 的扩展域内计算影响系
数矩阵 $\boldsymbol{K}$, 其中矩阵中心元素表示坐标原点。

(3) 分别对 $\boldsymbol{p}_{\text {new }}$ 和 $\boldsymbol{K}$ 进行傅里叶变换, 得到 $\operatorname{FFT}\left[\boldsymbol{p}_{\text {new }}\right]$ 和 $\operatorname{FFF}[\boldsymbol{K}]$ 。

(4) 对 FFT $\left[\boldsymbol{p}_{\text {new }}\right]$ 和 FFF $[\boldsymbol{K}]$ 进行点乘运算, 并对 其乘积进行逆傅里叶变换可以得到: $\boldsymbol{u}_{n e w}=\operatorname{IFFT}\left[\operatorname{FFT}\left[\boldsymbol{p}_{n e w}\right] \cdot \operatorname{FFF}[\boldsymbol{K}]\right]$ 。

(5) 取 $u^{\alpha, \beta}=u_{\text {new }}^{\alpha+M-1, \beta+N-1}$, 即可得到大小为 $M \times$ $N$ 的目标计算区域的弹性变形矩阵 $\boldsymbol{u}$ 。

\section{3 弹塑性粗粗表面的实际接触面积演变规律}

为了探究上述三个因素对实际接触面积演变规 律的影响，本节设计了正交试验对具有不同参数类 型的弹塑性粗楉表面与刚性平面的接触问题进行数 值计算，正交试验的因素和水平如表 1 所示。对于 每一种参数类型, 分别建立了 5 个弹塑性接触对, 并使用第 1.2 节所述的数值计算建模方法对它们的 接触行为进行求解。受模型的计算效率和收敛性的 限制，只对各个接触对 $0 \%$ ～30\%的实际接触面积范 围的接触状态进行求解。如图 3 所示，由于每种参 数类型的 5 个接触对的实际接触面积的标准差均小 于其均值的 $1 \%$, 每一种参数类型的 5 个接触对的实 际接触面积演变规律之间的差异基本可以忽略不 计。因此, 本节将每种参数类型的 5 个接触对的实 际接触面积均值作为该参数类型的所有接触对的实 际接触面积。

\begin{tabular}{|c|c|c|c|c|c|}
\hline \multirow{2}{*}{ 因素 } & \multicolumn{5}{|c|}{ 水平 } \\
\hline & 1 & 2 & 3 & 4 & 5 \\
\hline 表面梯度均方根 & 0.00408 & 0.00807 & 0.161 & 0.335 & 1.21 \\
\hline $\begin{array}{c}\text { 等效弹性模量 } \\
\qquad / \mathrm{GPa}\end{array}$ & 10 & 50 & 100 & - & - \\
\hline 屈服强度/MPa & 93.33 & 466.67 & 933.33 & - & - \\
\hline
\end{tabular}

表 1 弹塑性接触对的参数类型列表

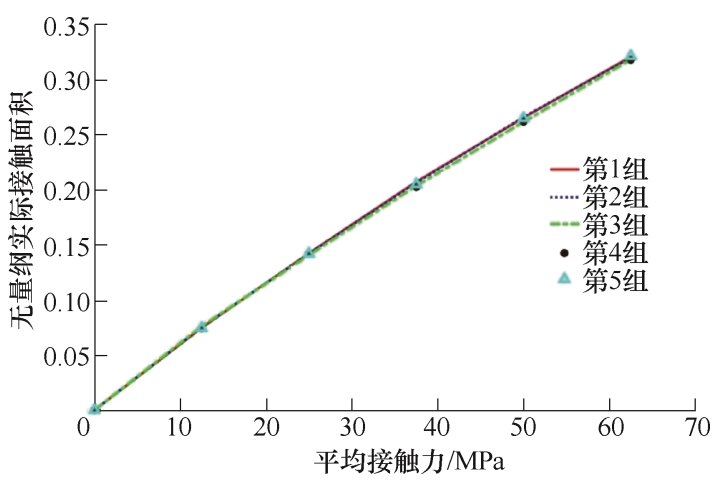

图 3 参数类型 1 的 5 组接触对的实际接触面积变化曲线

对计算结果进行分析发现, 当实际接触面积 从 $0 \%$ 增加到 $30 \%$ 时, 所有参数类型的弹塑性粗 䊁表面的实际接触面积都近似随着平均接触压力 
线性增加 (实际接触面积与平均接触压力近似成 正比例关系)。使用最小二乘法线性拟合实际接触 面积-平均接触压力曲线, 得到比例系数 $K$ 如表 2 所示。通过对比表中数据可以发现, 比例系数 $K$ 随着屈服强度的增加而降低; 但是参数类型 2 和 3 的结果似乎不遵循这个规律(它们的比例系数 $K$ 相等), 这是由于这两种参数类型的接触对塑性接
触区域所占比例很低所导致的(塑性系数很小)。 同时, 比例系数 $K$ 总体上随着弹性模量的增加而 降低、随着表面梯度的均方根的增加而降低。定 义 $\omega$ 为无量纲比例系数, 且 $\omega=K \cdot H$ 。结合硬度 与屈服强度的关系以及塑性指数的定义, 计算出 每一个接触对的无量纲比例系数 $\omega$ 和塑性指数 $\psi$ 如表 2 所示。

表 2 不同参数类型弹塑性接触对的计算结果

\begin{tabular}{|c|c|c|c|c|c|c|c|}
\hline 编号 & 比例系数 $K$ & 无量纲比例系数 $\omega$ & 塑性指数 $\psi$ & 编号 & 比例系数 $K$ & 无量纲比例系数 $\omega$ & 塑性指数 $\psi$ \\
\hline 1 & $4.93 \times 10^{-3}$ & 1.38 & 1.46 & 24 & $4.06 \times 10^{-4}$ & 1.14 & 2.87 \\
\hline 2 & $3.72 \times 10^{-3}$ & 5.21 & 0.29 & 25 & $3.56 \times 10^{-3}$ & 1.00 & 57.45 \\
\hline 3 & $3.72 \times 10^{-3}$ & 10.41 & 0.15 & 26 & $7.26 \times 10^{-4}$ & 1.02 & 11.48 \\
\hline 4 & $3.71 \times 10^{-3}$ & 1.04 & 7.29 & 27 & $3.97 \times 10^{-4}$ & 1.11 & 5.74 \\
\hline 5 & $9.94 \times 10^{-4}$ & 1.39 & 1.46 & 28 & $3.63 \times 10^{-3}$ & 1.02 & 11.95 \\
\hline 6 & $8.07 \times 10^{-4}$ & 2.26 & 0.73 & 29 & $8.31 \times 10^{-4}$ & 1.16 & 2.39 \\
\hline 7 & $3.82 \times 10^{-3}$ & 1.07 & 14.58 & 30 & $5.48 \times 10^{-4}$ & 1.53 & 1.20 \\
\hline 8 & $8.09 \times 10^{-4}$ & 1.13 & 2.91 & 31 & $3.57 \times 10^{-3}$ & 1.00 & 59.75 \\
\hline 9 & $5.69 \times 10^{-4}$ & 1.59 & 1.46 & 32 & $7.40 \times 10^{-4}$ & 1.04 & 11.94 \\
\hline 10 & $4.05 \times 10^{-3}$ & 1.13 & 2.88 & 33 & $3.73 \times 10^{-4}$ & 1.04 & 5.98 \\
\hline 11 & $1.87 \times 10^{-3}$ & 2.62 & 0.58 & 34 & $3.56 \times 10^{-3}$ & 1.00 & 119.51 \\
\hline 12 & $1.85 \times 10^{-3}$ & 5.18 & 0.29 & 35 & $7.30 \times 10^{-4}$ & 1.02 & 23.88 \\
\hline 13 & $3.64 \times 10^{-3}$ & 1.02 & 14.42 & 36 & $3.63 \times 10^{-4}$ & 1.02 & 11.95 \\
\hline 14 & $8.05 \times 10^{-4}$ & 1.13 & 2.88 & 37 & $3.57 \times 10^{-3}$ & 1.00 & 43.18 \\
\hline 15 & $4.95 \times 10^{-4}$ & 1.39 & 1.44 & 38 & $7.36 \times 10^{-4}$ & 1.03 & 8.63 \\
\hline 16 & $3.58 \times 10^{-3}$ & 1.00 & 28.83 & 39 & $3.84 \times 10^{-4}$ & 1.07 & 4.32 \\
\hline 17 & $7.52 \times 10^{-4}$ & 1.05 & 5.76 & 40 & $3.55 \times 10^{-3}$ & 0.99 & 215.92 \\
\hline 18 & $4.06 \times 10^{-4}$ & 1.14 & 2.88 & 41 & $7.13 \times 10^{-4}$ & 1.00 & 43.14 \\
\hline 19 & $3.75 \times 10^{-3}$ & 1.05 & 5.74 & 42 & $3.59 \times 10^{-4}$ & 1.00 & 21.59 \\
\hline 20 & $1.09 \times 10^{-3}$ & 1.53 & 1.15 & 43 & $3.55 \times 10^{-3}$ & 0.99 & 431.83 \\
\hline 21 & $9.35 \times 10^{-4}$ & 2.62 & 0.57 & 44 & $7.11 \times 10^{-4}$ & 1.00 & 86.27 \\
\hline 22 & $3.58 \times 10^{-3}$ & 1.00 & 28.72 & 45 & $3.57 \times 10^{-4}$ & 1.00 & 43.18 \\
\hline 23 & $7.78 \times 10^{-4}$ & 1.09 & 5.74 & & & & \\
\hline
\end{tabular}

无量纲比例系数 $\omega$ 随塑性指数 $\psi$ 的变化如图 4 所示(为了便于绘图和观察, 该图采用了双对数坐 标)。 $\omega$ 随 $\psi$ 的变化可以近似用图中曲线进行拟合, 该曲线的关系式为

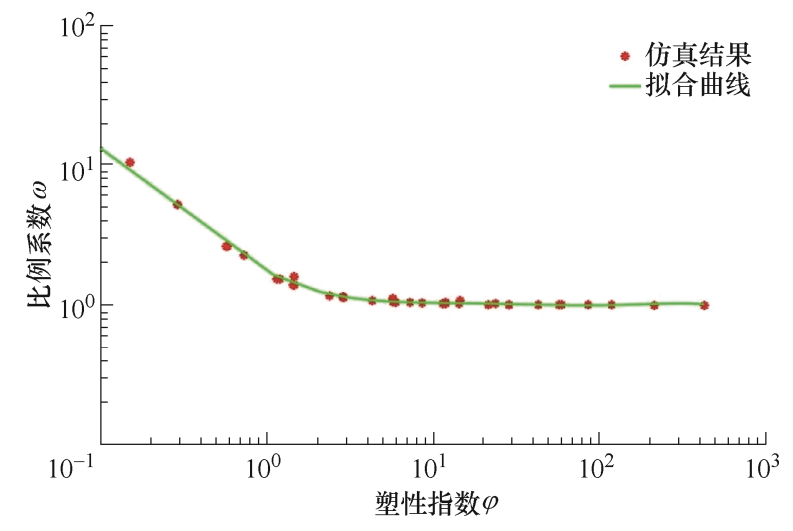

图 4 无量纲比例系数 $\omega$ 随塑性指数 $\psi$ 的变化曲线

$$
\begin{aligned}
\lg \omega= & -0.0169 \times(\lg \psi)^{6}+0.1108 \times(\lg \psi)^{5}- \\
& 0.2108 \times(\lg \psi)^{4}-0.0385 \times(\lg \psi)^{3}+ \\
& 0.5556 \times(\lg \psi)^{2}-0.6237 \times \lg \psi+0.2382
\end{aligned}
$$

结合式(13)以及 $\omega$ 的定义, 即可近似得到弹塑 性粗楉表面受压接触时的实际接触面积随着平均接 触压力变化的关系式为

$$
a=\frac{\bar{p}}{H} 10^{A}
$$

$$
\begin{aligned}
& A=\lg \omega=-0.0169(\lg \psi)^{6}+0.1108(\lg \psi)^{5}- \\
& 0.2108(\lg \psi)^{4}-0.0385(\lg \psi)^{3}+ \\
& 0.5556(\lg \psi)^{2}-0.6237 \lg \psi+0.2382
\end{aligned}
$$




\section{2 试验验证}

为了验证本文所总结的弹塑性粗粘表面实际接 触面积演变规律, 使用式(13)对由如图 5 所示的各 向同性随机粗䊁表面所组成的接触对的弹塑性接触 问题进行求解, 并将计算结果与试验测量 ${ }^{[60-61]}$ 和有 限元仿真结果 ${ }^{[61]}$ 进行了对比研究。该接触对的等效 弹性模量为 $34066 \mathrm{MPa}$, 表面硬度为 $310 \mathrm{MPa}$, 表 面梯度的均方根为 0.7109 。表 3 将本文计算的比例 系数 $K$ 与试验测量结果和有限元仿真结果进行了对 比, 本文计算结果与试验测量和有限元仿真结果的 相对误差均可忽略不计, 证明了本文所总结的弹塑 性粗䊁表面实际接触面积演变规律的准确性。
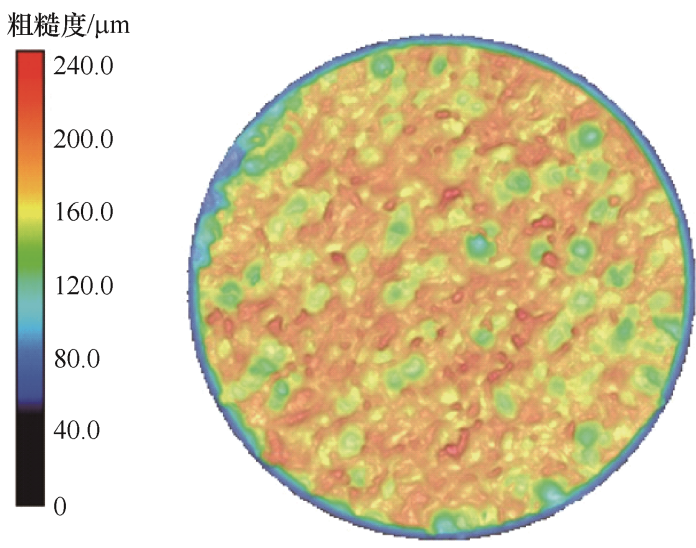

图 5 各向同性随机粗糙表面

表 3 本文计算结果与试验测量和有限元仿真结果的对比

\begin{tabular}{cccc}
\hline & 本文计算 & 试验测量 & 有限元仿真 \\
\hline 比例系数 $K$ & 0.00320 & 0.00331 & 0.00316 \\
相对误差 $(\%)$ & - & 3.32 & 1.27 \\
\hline
\end{tabular}

\section{3 结论}

（1）针对弹塑性粗粮表面实际接触面积的演变 规律尚不明确的问题，结合多微凸体模型、Persson 理论、塑性接触理论和塑性指数的研究得出结论: 影响弹塑性粗糙表面接触状态的主要因素为材料的 等效弹性模量、硬度(或屈服强度)和表面梯度的均 方根。

（2）在此基础上，通过对多组弹塑性粗粘表面 的接触行为进行数值仿真计算, 发现当实际接触面 积小于 $30 \%$ 时, 弹塑性粗糙表面的实际接触面积与 平均接触压力近似成正比例关系, 揭示了该正比例 系数与材料的弹性模量、屈服强度和表面梯度的均
方根之间的映射关系, 并最终得到实际接触面积的 计算公式, 为弹塑性接触力学的后续工程应用奠定 了基础。

\section{参 考 文 献}

[1] HERTZ H. On the contact of elastic solids[J]. Journal Reine. Angew. Math., 1881, 92(1): 156-171.

[2] GREENWOOD J A, WILLIAMSON J B P. Contact of nominally flat surfaces[J]. Proceedings of the Royal Society of London, 1966, 295(1442): 300-319.

[3] GREENWOOD J A, TRIPP J H. The elastic contact of rough spheres[J]. Journal of Applied Mechanics, 1967, 34(1): 153-160.

[4] HISAKADO T. Effect of surface roughness on contact between solid surfaces[J]. Wear, 1974, 28(2): 217-234.

[5] GREENWOOD J A. The contact of two nominally flat rough surfaces[J]. Proceedings of the Institution of Mechanical Engineers, 1970, 185(1970): 625-634.

[6] BUSH A W, GIBSON R D, KEOGH G P. Strongly anisotropic rough surfaces[J]. Journal of Tribology, 1979, 101(1): 15-21.

[7] CHANG W R, ETSION I, BOGY D B. An elastic-plastic model for the contact of rough surfaces[J]. Journal of Tribology, 1987, 109(2): 257-263.

[8] ZHAO Y, MAIETTA D M, CHANG L. An asperity microcontact model incorporating the transition from elastic deformation to fully plastic flow[J]. Journal of Tribology, 2000, 122(1): 86-93.

[9] ZHAO Y, CHANG L. A model of asperity interactions in elastic-plastic contact of rough surfaces[J]. Journal of Tribology, 2001, 123(4): 857-864.

[10] KOGUT L, ETSION I. Elastic-plastic contact analysis of a sphere and a rigid flat[J]. Journal of Applied Mechanics, 2002, 69(5): 657-62.

[11] JACKSON R L, GREEN I. A finite element study of elasto-plastic hemispherical contact against a rigid flat[J]. Journal of Tribology, 2005, 127(2): 343-54.

[12] WADWALKAR S S, JACKSON R L, KOGUT L. A study of the elastic-plastic deformation of heavily deformed spherical contacts[J]. ARCHIVE Proceedings of the Institution of Mechanical Engineers Part J Journal of Engineering Tribology, 2010, 208-210(1): 1-12.

[13] YASTREBOV V A, ANCIAUX G, MOLINARI J F. From infinitesimal to full contact between rough surfaces: Evolution of the contact area[J]. International Journal of Solids \& Structures, 2015, 52: 83-102.

[14] PERSSON B N. Elastoplastic contact between randomly rough surfaces[J]. Phys. Rev. Lett., 2001, 87(11): 116101. 
[15] PERSSON B N, BUCHER F, CHIAIA B. Elastic contact between randomly rough surfaces: Comparison of theory with numerical results[J]. Physical Review B, 2002, 65(18): 184106.

[16] CARBONE G, BOTTIGLIONE F. Asperity contact theories: Do they predict linearity between contact area and load? [J]. Journal of the Mechanics \& Physics of Solids, 2008, 56(8): 2555-2572.

[17] YASTREBOV VA, ANCIAUX G, MOLINARI J F. The role of the roughness spectral breadth in elastic contact of rough surfaces[J]. Journal of the Mechanics \& Physics of Solids, 2017, 107: 469-493.

[18] HU Y Z, BARBER G, DONG Z. Numerical analysis for the elastic contact of real rough surfaces[J]. Tribology Transactions, 1999, 42(3): 443-452.

[19] STANLEY H M, KATO T. An FFT-based method for rough surface contact[J]. Journal of Tribology, 1997, 119(3): 481-485.

[20] LI J, BERGER E J. A semi-analytical approach to three-dimensional normal contact problems with friction[J]. Computational Mechanics, 2003, 30(4): 310-322.

[21] POHRT R, LI Q. Complete boundary element formulation for normal and tangential contact problems[J]. Physical Mesomechanics, 2014, 17(4): 334-340.

[22] CARBONE G, PUTIGNANO C. A novel methodology to predict sliding and rolling friction of viscoelastic materials: Theory and experiments[J]. Journal of the Mechanics \& Physics of Solids, 2013, 61(8): 1822-1834.

[23] CHEN W W, LIU S, WANG Q J. Fast Fourier transform based numerical methods for elasto-plastic contacts of nominally flat surfaces[J]. Journal of Applied Mechanics, 2008, 75(1): 011022.

[24] WANG W Z, WANG Z J, WANG W Z, et al. A numerical elastic-plastic contact model for rough surfaces[J]. Tribology Transactions, 2010, 53(2): 224-238.

[25] WRIGGERS P. Computational contact mechanics[J]. Computational Mechanics, 2003， 32(1): 141.

[26] JACQ C, NE' LIAS D, LORMAND G, et al. Development of a three-dimensional semi-analytical elastic-plastic contact code[J]. Journal of Tribology, 2002, 124(4): 653-667.

[27] BOUCLY V, NE' LIAS D, LIU S, et al. Contact analyses for bodies with frictional heating and plastic behavior[J]. Journal of Tribology, 2005， 127(2): 355-364.

[28] NELIAS D, BOUCLY V, BRUNET M. Elastic-plastic contact between rough surfaces: Proposal for a wear or running-in model[J]. Journal of Tribology, 2006, 128: 236-244.
[29] NéliAS D, ANTALUCA E, BOUCLY V, et al. A three-dimensional semi-analytical model for elastic-plastic sliding contacts[J]. Journal of Tribology, 2007, 129: 761-771.

[30] CHEN W W, WANG Q J. Thermomechanical analysis of elastoplastic bodies in a sliding spherical contact and the effects of sliding speed, heat partition, and thermal softening[J]. Journal of Tribology, 2008, 130(4): 041402.

[31] WANG Z J, WANG W Z, HU Y Z, et al. A numerical elastic-plastic contact model for rough surfaces[J]. Tribology Transactions, 2010, 53(2): 224-238.

[32] ZHOU K, CHEN W W, KEER L M, et al. A fast method for solving three-dimensional arbitrarily shaped inclusions in a half space[J]. Computer Methods in Applied Mechanics and Engineering, 2009, 198(9-12): 885-892.

[33] LIU S, WANG Q. Elastic fields due to eigenstrains in a half-space[J]. Journal of Applied Mechanics，2005， 72: 871-878

[34] LIU S, JIN X, WANG Z, et al. Analytical solution for elastic fields caused by eigenstrains in a half-space and numerical implementation based on FFT[J]. International Journal of Plasticity, 2012, 35: 135-154.

[35] KOMVOPOULOS K, CHOI D H. Elastic finite element analysis of multi-asperity contacts[J]. Journal of Tribology, 1992, 114(4): 823-831.

[36] BORRI-BRUNETTO M, CARPINTERI A, CHIAIA B. Lacunarity of the contact domain between elastic bodies with rough boundaries[M]. Netherlands: Springer, 1998.

[37] HYUN S, PEI L, MOLINARI J F, et al. Finite-element analysis of contact between elastic self-affine surfaces[J]. Phys. Rev. E Stat. Nonlin. Soft. Matter. Phys., 2004, 70(2): 026117.

[38] HYUN S, ROBBINS M O. Elastic contact between rough surfaces : Effect of roughness at large and small wavelengths[J]. Tribology International, 2007， 40(10): 1413-1422.

[39] PEI L, HYUN S, MOLINARI J F, et al. Finite element modeling of elasto-plastic contact between rough surfaces[J]. Journal of the Mechanics \& Physics of Solids, 2005, 53(11): 2385-2409.

[40] SAHOO P, GHOSH N. Finite element contact analysis of fractal surfaces[J]. Journal of Physics D Applied Physics, 2007, 40(14): 4245.

[41] WALTER C, MITTERER C. 3D versus 2D finite element simulation of the effect of surface roughness on nanoindentation of hard coatings[J]. Surface \& Coatings Technology, 2009, 203(20): 3286-3290.

[42] YASTREBOV V A, DURAND J, PROUDHON H, et al. Rough surface contact analysis by means of the finite 
element method and of a new reduced model[J].

Mechanical Reports, 2011, 339(7): 473-490.

[43] BRYANT M J, EVANS H P, SNIDLE R W. Plastic deformation in rough surface line contacts-a finite element study[J]. Tribology International, 2012, 46(1): 269-278.

[44] WRIGGERS P, REINELT J. Multi-scale approach for frictional contact of elastomers on rough rigid surfaces[J]. Computer Methods in Applied Mechanics \& Engineering, 2010, 198(21): 1996-2008.

[45] ZHANG F, LIU J, DING X, et al. An approach to calculate leak channels and leak rates between metallic sealing surfaces[J]. Journal of Tribology，2017，139(1): 011708-18.

[46] YAN W, KOMVOPOULOS K. Contact analysis of elastic-plastic fractal surfaces[J]. Journal of Applied Physics, 1998, 84(7): 3617.

[47] GAO Y F, BOWER A F. Elastic-plastic contact of a rough surface with Weierstrass profile[J]. Proceedings Mathematical Physical \& Engineering Sciences, 2006, 462(2065): 319-348.

[48] BUSH A W, GIBSON R D, THOMAS T R. The elastic contact of a rough surface[J]. Wear, 1975, 35(1): 87-111.

[49] GREENWOOD J A. A simplified elliptic model of rough surface contact[J]. Wear, 2006, 261(2): 191-200.

[50] TABOR D. The hardness of metals[M]. London: Oxford University Press, 1951.

[51] RICHARDS T H. Energy methods in stress analysis: With an introduction to finite element techniques[M]. New York: Halsted Press, 1977.

[52] TIAN X, BHUSHAN B. A numerical three-dimensional model for the contact of rough surfaces by variational principle[J]. Journal of Tribology, 1996, 1118(1): 33-42.

[53] JOHNSON K L. Contact mechanics[M]. Cambridge: Cambridge University Press, 1985.

[54] VENNER C H, LUBRECHT A A. Multi-level methods in lubrication[M]. New York: Elsevier, 2000.

[55] SAINSOT P, LUBRECHT A A. Efficient solution of the dry contact of rough surfaces: A comparison of fast
Fourier transform and multigrid methods[J]. Proc. Inst. Mech. Eng. Part J-J. Eng. Tribol., 2011, 225(J6): 441-448.

[56] 王文中. 混合润滑的数值模拟和实验研究 [D]. 北京: 清 华大学, 2003.

WANG Wenzhong. Numerical simulation and experimental study of mixed lubrication [D]. Beijing: Tsinghua University, 2003.

[57] 王文中, 胡元中, 王慧. 基于快速傅里叶变换和共轭梯 度法求解干接触问题 [J]. 机械工程学报, 2006, 42(7): 14-18.

WANG Wenzhong, HU Yuanzhong, WANG Hui. Solving dry contact problems based on fast Fourier transform and conjugate gradient method [J]. Journal of Mechanical Engineering, 2006, 42(7): 14-18.

[58] JU Y, FARRIS T. Spectral analysis of two-dimensional contact problems[J]. Journal of Tribology, 1996, 118: 320-328.

[59] WANG W, WANG H, LIU Y, et al. A comparative study of the methods for calculation of surface elastic deformation $[\mathrm{J}]$. Proceedings of the Institution of Mechanical Engineers, Part J: Journal of Engineering Tribology, 2003, 217(2): 145-154.

[60] ZHANG F, LIU J, DING X, et al. A discussion on the capability of x-ray computed tomography for contact mechanics investigations[J]. Tribology International, 2020, 145: 106167

[61] ZHANG F, LIU J, DING X, et al. Experimental and finite element analyses of contact behaviors between non-transparent rough surfaces[J]. Journal of the Mechanics and Physics of Solids, 2019, 126: 87-100.

作者简介: 刘检华, 男, 1977 年出生, 博士, 教授, 博士研究生导师。 主要研究方向为产品装配与检测技术。

E-mail: jeffliu@bit.edu.cn

张飞凯, 男, 1993 年出生, 博士。主要研究方向为接触力学、静密封。 E-mail: zhangfkbit@163.com

丁晓宇(通信作者), 男, 1984 年出生，博士，副教授，硕士研究生导师。 主要研究方向为基于机器视觉的装配精度测量、基于接触力学的装配变 形预测、基于数字孪生的装配精度在线跟踪、基于信号处理和机器学习 的装配质量控制等。

E-mail: xiaoyu.ding@bit.edu.cn 